TRANSACTIONS OF THE

AMERICAN MATHEMATICAL SOCIETY

Volume 351, Number 4, April 1999, Pages 1391-1401

S 0002-9947(99)02061-9

\title{
EIGENVALUE ESTIMATE ON COMPLETE NONCOMPACT RIEMANNIAN MANIFOLDS AND APPLICATIONS
}

\author{
MANFREDO P. DO CARMO AND DETANG ZHOU
}

\begin{abstract}
We obtain some sharp estimates on the first eigenvalues of complete noncompact Riemannian manifolds under assumptions of volume growth. Using these estimates we study hypersurfaces with constant mean curvature and give some estimates on the mean curvatures.
\end{abstract}

\section{INTRODUCTION}

Let $M$ be an $n$-dimensional complete Riemannian manifold and let $\Delta$ be Laplacian operator on $M$ (with the convention $\Delta=\frac{d^{2}}{d x^{2}}$ on $R^{1}$ ). We recall that the first eigenvalue $\lambda_{1}(D)$ for some bounded open domain in $M$ is defined as the smallest $\lambda$ that satisfies

$$
\Delta u+\lambda u=0 \text { in } D
$$

for some non-zero function $u$ on $M$ with $\left.u\right|_{\partial D}=0$. The first eigenvalue of $M$ is defined by

$$
\lambda_{1}(M)=\inf \left\{\lambda_{1}(D): \quad D \subset M \text { is a bounded open domain }\right\} .
$$

Let $\Omega \subset M$ be a subset of $M$. The first eigenvalue of $M \backslash \Omega$ is defined by

$$
\lambda_{1}(M \backslash \Omega)=\inf \left\{\lambda_{1}(D): \quad D \subset M \backslash \Omega \text { is a bounded open domain }\right\} .
$$

It is well known that $\lambda_{1}\left(R^{n}\right)=\lambda_{1}\left(R^{n} \backslash \Omega\right)=0$ for any compact set in $R^{n}$. Cheng and Yau [CY, Proposition 9] showed that $\lambda_{1}(M)=0$ if the manifold has polynomial volume growth. But Cheng-Yau's result doesn't hold in the case of a manifold punctured by a compact subset. That is to say, in general, $\lambda_{1}(M) \neq \lambda_{1}(M \backslash \Omega)$ if $\Omega \neq \emptyset$, and this can be seen from the following example

Example. Let $M=\left(R^{2}, d s^{2}\right)$, with $d s^{2}=d r^{2}+g^{2}(r) d \theta^{2}$, where $g(r)$ is a nonnegative $C^{2}$ function on $[0,+\infty)$ with $g(0)=0$ and $g(r)>0$ for all $r>0$ and $g(r)=e^{-r}$ when $r>1$. It is easy to see that the volume of $M$ is finite and thus $\lambda_{1}(M)=0$. But we have that $u(x)=e^{-\frac{1}{2}|x|}$ is a positive solution of

$$
\Delta u+\lambda u=0
$$

on $M \backslash B(1)$ where $B(1)$ is the unit ball and $\lambda=\frac{1}{4}$. Then $\lambda_{1}(M \backslash B(1)) \geq \frac{1}{4}$. To see this, we need to notice the fact $[\mathrm{CY}]$ that $\lambda_{1}(M \backslash \Omega) \geq \inf \left(-\frac{\Delta u}{u}\right)$ for any positive $C^{2}$ function $u$ on $M \backslash \Omega$.

Received by the editors November 15, 1996 and, in revised form, February 28, 1997. 1991 Mathematics Subject Classification. Primary 53C42; Secondary 53A10, 53C20, 35J60.

Key words and phrases. Riemannian manifold, eigenvalue, hypersurface, mean curvature. Supported partially by NNSFC and TWAS-IMPA membership.

(C)1999 American Mathematical Society 
It is then a natural question to estimate $\lambda_{1}(M \backslash \Omega)$ and $\lambda_{1}(M)$ under various volume growth conditions; for instance, can we give an estimate for $\lambda_{1}(M)$ when the volume of the manifold grows exponentially? From the geometric viewpoint it is often necessary to give an estimate of $\lambda_{1}(M \backslash \Omega)$. The problem arises naturally in the study of unstable hypersurfaces with constant mean curvatures. In order to explain some of our results we give some definitions here. Let $x: M^{n} \rightarrow \bar{M}^{n+1}$ be an isometric immersion of a complete, noncompact Riemannian $n$-dimensional manifold $M^{n}$ into an oriented, complete, Riemannian $(n+1)$-dimensional manifold, $N$ a smooth unit normal field along $M$, and $\overline{\operatorname{Ric}}(N)$ the value of the Ricci curvature of $\bar{M}^{n+1}$ in the vector $N$. Here $\overline{\operatorname{Ric}}(N)=\sum_{i=1}^{n} K\left(e_{i} \wedge N\right)$ (this is different from the normalized one). The index ind $M$ of $\mathrm{M}$ is defined as follows. Let $L$ be the second order differential operator on $M$ given by

$$
L=\Delta+\|B\|^{2}+\overline{\operatorname{Ric}}(N),
$$

where $\|B\|^{2}$ is the second fundamental form of $x$. Associated to $L$ is the quadratic form

$$
I(f)=-\int_{M} f L f d M
$$

defined on the vector space of functions $f$ on $M$ that have support on a compact domain $K \subset M$. For each such $K$, define the index $\operatorname{ind}_{L} K$ of $L$ in $K$ as the maximal dimension of a subspace where $I$ is negative definite. The index ind $M$ of $L$ in $M$ is the number defined by

$$
\text { ind } M=\sup _{K \subset M} \operatorname{ind}_{L} K
$$

where the supremum is taken over all compact domains $K \subset M$.

In this paper, in section 2, we give a new oscillation result for a kind of second order ordinary differential equation which is interesting for us. Using this, we obtain some estimates of first eigenvalue on manifolds in section 3 ; we prove that for example, $\lambda_{1}(M) \leq \frac{a^{2}}{4}$ if the volume of ball $B(r)$ is bounded above by $C e^{a r}$ for some positive constants $C$, and $a$. That is to say: the usual curvature conditions for estimating $\lambda_{1}$ can replaced by volume conditions which are weaker and more general. Then in section 4 we give some applications to submanifolds. We assume that $\operatorname{vol}(M)=+\infty$ and show that: If $x$ has constant mean curvature $H$, ind $M<$ $+\infty$ and the volume of $M$ has subexponential growth, then there exists a positive constant $r_{0}>0$ such that for all $r \geq r_{0}$

$$
H^{2} \leq-\frac{1}{n} \inf _{M \backslash B(r)} \overline{\operatorname{Ric}}(N),
$$

and thus $x$ has to be minimal if $\bar{M}^{n+1}$ has nonnegative Ricci curvature. If the volume of $M$ satisfies $\operatorname{vol}(B(r)) \leq C e^{a r}$ when $r \geq 0$ for some positive constants $C$ and $a$, then there exists a positive constant $r_{0}>0$ such that for all $r \geq r_{0}$

$$
H^{2} \leq \frac{a^{2}}{4 n}-\frac{1}{n} \inf _{M \backslash B(r)} \overline{\operatorname{Ric}}(N) .
$$

We also can use our results to give estimates for mean curvatures in higher codimension. 


\section{ACKNOWLEDGEMENTS}

This work was done while the second author was visiting Instituto de Matemática Pura e Aplicada by an associate membership scheme of TWAS-IMPA. He wishes to thank these institutions for support and IMPA for the hospitality. The authors want to thank the referee for calling their attention to the reference $[\mathrm{T}]$ which contains our Corollary 3.3.

\section{Oscillation theorem}

We are here concerned with the oscillatory behavior of solutions of the following second order ordinary differential equation:

$$
\left(v(t) x^{\prime}(t)\right)^{\prime}+\lambda v(t) x(t)=0, \quad t \geq T_{0},
$$

where $v(t)$ is a positive continuous function on $\left[T_{0},+\infty\right)$ and $\lambda$ is a positive constant. We call, as usual, equation (2.1) oscillatory if all solutions of (2.1) have arbitrary large zeroes on $[T,+\infty)$; otherwise, we say equation (2.1) is nonoscillatory. The literature on second order linear oscillation is voluminous (for references see, for example, $[\mathrm{W}]$ and references therein). We will give an oscillation criteria in integral form. There have been many criteria for oscillations of (2.1) in more general form, but we didn't find the result which is useful in our discussions below.

Theorem 2.1. Assume $v(t)$ is a positive continuous function on $\left[T_{0},+\infty\right)$ and $\int^{+\infty} v(\tau) d \tau=+\infty$. Then equation (2.1) is oscillatory provided either

(a) $\lambda>0$ is a constant and $V(t)=\int_{T_{0}}^{t} v(\tau) d \tau \leq C t^{a}$ for some positive constants $C$ and $a$, or

(b) $\lambda>\frac{a^{2}}{4}$ is a constant and $V(t)=\int_{T_{0}}^{t} v(\tau) d \tau \leq C e^{a t}$ for some positive constants $C$ and $a$.

Proof. Assume for the sake of contradiction that (2.1) is nonoscillatory; then there exist a solution $x(t)$ of $(2.1)$ and a positive constant $T>T_{0}$ such that $x(t)>0$ for any $t \geq T$. Now we set

$$
y(t)=-\frac{v(t) x^{\prime}(t)}{x(t)}
$$

Then $y(t)$ is well defined on $[T,+\infty)$ and satisfies the following Riccati equation:

$$
y^{\prime}(t)=\frac{y^{2}(t)}{v(t)}+\lambda v(t)
$$

So $y^{\prime}(t)>0$, and $y(t)$ is an increasing function on $[T,+\infty)$. We need to divide the rest of the proof into two cases:

(i) $\int_{T}^{+\infty} \frac{d \tau}{v(\tau)}<+\infty$. In this case we know (2.3) implies $y^{\prime}(t) \geq \lambda v(t)$, so $y(t) \geq$ $\lambda(V(t)-V(T))+y(T)$ is eventually positive, and we may assume $y(t)>0$, for any $t \geq T$. By (2.3) we have, for any $t \geq T$

$$
\begin{aligned}
y^{\prime}(t) & \geq 2 \sqrt{\lambda} y(t), \\
\frac{y^{\prime}(t)}{y(t)} & \geq 2 \sqrt{\lambda}, \\
\log \frac{y(t)}{y(T)} & \geq 2 \sqrt{\lambda}(t-T), \\
y(t) & \geq y(T) e^{2 \sqrt{\lambda}(t-T) .}
\end{aligned}
$$


On the other hand,

$$
\begin{aligned}
\frac{y^{\prime}(t)}{y^{2}(t)} & \geq \frac{1}{v(t)} \\
-\frac{1}{y(t)}+\frac{1}{y(t-1)} & \geq \int_{t-1}^{t} \frac{d \tau}{v(\tau)} \\
\frac{1}{y(t)} & \leq \frac{1}{y(t-1)}-\int_{t-1}^{t} \frac{d \tau}{v(\tau)} \\
& \leq \frac{1}{y(T) e^{2 \sqrt{\lambda}(t-T-1)}}-\int_{t-1}^{t} \frac{d \tau}{v(\tau)}
\end{aligned}
$$

We have

$$
\begin{aligned}
1 & =\int_{t-1}^{t} d \tau \leq\left(\int_{t-1}^{t} \frac{d \tau}{v(\tau)}\right)^{\frac{1}{2}} \cdot\left(\int_{t-1}^{t} v(\tau) d \tau\right)^{\frac{1}{2}} \\
& \leq\left(\int_{t-1}^{t} \frac{d \tau}{v(\tau)}\right)^{\frac{1}{2}} \cdot(V(t))^{\frac{1}{2}} .
\end{aligned}
$$

So

$$
\int_{t-1}^{t} \frac{1}{v(\tau)} d \tau \geq \frac{1}{V(t)}
$$

If (a) is satisfied, we have from (2.4), when $t$ is large enough,

$$
\begin{aligned}
0<\frac{1}{y(t)} & \leq \frac{1}{y(T) e^{2 \sqrt{\lambda}(t-T-1)}}-\frac{1}{V(t)} \\
& \leq \frac{1}{y(T) e^{2 \sqrt{\lambda}(t-T-1)}}-\frac{1}{C t^{a}}<0 .
\end{aligned}
$$

Thus we arrive at a contradiction.

If (b) is satisfied, (2.4) implies, when $t$ is large enough,

$$
\begin{aligned}
0<\frac{1}{y(t)} & \leq \frac{1}{y(T) e^{2 \sqrt{\lambda}(t-T-1)}-\frac{1}{C e^{a t}}} \\
& \leq\left[\frac{C}{y(T) e^{(2 \sqrt{\lambda}-a) t-2 \sqrt{\lambda}(T+1))}}-1\right] \frac{1}{C e^{a t}}<0 .
\end{aligned}
$$

where we used the condition $\lambda>\frac{a^{2}}{4}$. We also get a contradiction.

(ii) $\int_{T}^{+\infty} \frac{d \tau}{v(\tau)}=+\infty$. Let

$$
s(t)=\int_{T}^{t} \frac{d \tau}{v(\tau)}
$$

Then $s(t) \rightarrow+\infty$ as $t \rightarrow+\infty$, and

$$
\frac{d x}{d t}=\frac{d x}{d s} \cdot \frac{d s}{d t}=\frac{d x}{d s} \cdot \frac{1}{v(t)} .
$$

So,

$$
\frac{d}{d t}\left[v(t) x^{\prime}(t)\right]=\frac{d}{d s} \frac{d x}{d s} \cdot \frac{d s}{d t}=\frac{1}{v(t)} \cdot \frac{d^{2} x}{d s^{2}}
$$


and

$$
\frac{d^{2} x}{d s^{2}}+\lambda v^{2}(t) x(s)=0
$$

Now observe that

$$
\begin{aligned}
\int_{0}^{+\infty} v^{2}(t(s)) d s & =\int_{T}^{+\infty} v^{2}(t) \frac{d s}{d t} d t \\
& =\int_{T}^{+\infty} v(t) d t .
\end{aligned}
$$

If $\int_{T}^{+\infty} v(t) d t=+\infty$, from Fite [F, Theorem V] we know that (2.5) is oscillatory and then (2.1) is oscillatory. Thus we have proved our theorem.

Remark 2.1. The condition in (a) of Theorem 2.1 can be weakened to $V(t)$ grows subexponentially, in the sense that

$$
\lim _{t \rightarrow+\infty} \frac{\log V(t)}{t}=0 .
$$

The reason for this can be easily seen in the proof of part (b).

Remark 2.2. $\int^{+\infty} v(\tau) d \tau=+\infty$ is necessary in Theorem 2.1. If $v(t)=e^{-t}$, we can see that $x(t)=e^{\frac{1}{2} t}$ is a positive solution of $(2.1)$ on $[1,+\infty)$ with $\lambda=\frac{1}{4}$.

We prove here a theorem for the sake of completeness.

Theorem 2.2. If $v(t)$ is positive, then any solution of (2.1) with initial value

$$
x\left(T_{0}\right)=x_{0}, \text { and } x^{\prime}\left(T_{0}\right)=x_{1}
$$

can be extended to $\left[T_{0},+\infty\right)$.

Proof. Assume that $\left[t_{0}, T\right)$ is the maximal existence interval of (2.1) and (2.6) and $T$ is finite. Then $\lim _{t \rightarrow T}|x(t)|=+\infty$. We may assume $\lim _{t \rightarrow T} x(t)=+\infty$. Hence there exists $T_{1}$ such that $x(t)>0$, on $\left[T_{1}, T\right)$ and $x^{\prime}\left(T_{1}\right)>0$. Since

$$
\begin{aligned}
\left(v(t) x^{\prime}(t)\right)^{\prime} & =-\lambda v(t) x(t)<0, \\
x^{\prime}(t) & \leq \frac{v\left(T_{1}\right) x^{\prime}\left(T_{1}\right)}{v(t)}, \\
x(t) & \leq x\left(T_{1}\right)+\int_{T_{1}}^{t} \frac{v\left(T_{1}\right) x^{\prime}\left(T_{1}\right)}{v(s)} d s \\
& \leq x\left(T_{1}\right)+\int_{T_{1}}^{T} \frac{v\left(T_{1}\right) x^{\prime}\left(T_{1}\right)}{v(s)} d s<+\infty
\end{aligned}
$$

for any $t \in\left[T_{1}, T\right)$. So, $T=+\infty$.

\section{Eigenvalue estimates}

In this section we would like to use the oscillation result obtained in the previous section to give some estimates on the first eigenvalue of punctured manifolds. Fix a point $p \in M$ and denote by $B(r)=\{x \in M$, $\operatorname{dist}(x, p) \leq r\}$ and by $V(B(r))$ the volume of the ball $B(r)$. 
Theorem 3.1. Let $M$ be a complete and noncompact Riemannian manifold with infinite volume and $\Omega$ be an arbitrary compact subset of $M$. Then

(a) If $V(B(r)) \leq C r^{a}$ for any $r \geq r_{0}$ and some positive constants $C, r_{0}$ and $a$, then $\lambda_{1}(M \backslash \Omega)=0$.

(b) If $V(B(r)) \leq C e^{a r}$ for any $r \geq 0$ and some positive constants $C$ and $a$, then $\lambda_{1}(M \backslash \Omega) \leq \frac{a^{2}}{4}$.

Proof. Let $v(r)$ denote the area of sphere $\partial B(r)$. Then

$$
V(B(r))=\int_{0}^{r} v(t) d t
$$

If $V(M)=+\infty$, then $\int_{T}^{+\infty} v(t) d t=+\infty$ for any constant $T>0$. Since $\Omega$ is a compact subset, we can find a constant $T_{0}$ such that $\Omega \subset B\left(T_{0}\right)$.

If the condition in (a) is satisfied, then from Theorem 2.1 for any $\lambda>0$ there exists a nontrivial oscillatory solution $x_{\lambda}(t)$ of $(2.1)$ on $\left[T_{0},+\infty\right)$ with $v(t)$ defined here. Thus there exist two numbers $T_{1}^{\lambda}$ and $T_{2}^{\lambda}$ in $\left[T_{0},+\infty\right)$ such that $T_{1}^{\lambda}<T_{2}^{\lambda}$ and $x_{\lambda}\left(T_{1}^{\lambda}\right)=x_{\lambda}\left(T_{2}^{\lambda}\right)=0$, and $x_{\lambda}(t) \neq 0$ for any $t \in\left(T_{1}^{\lambda}, T_{2}^{\lambda}\right)$. Write $r(x)=\operatorname{dist}(x, p)$, $\varphi_{\lambda}(x)=x_{\lambda}(r(x))$ and $\Omega_{\lambda}=B\left(T_{2}^{\lambda}\right) \backslash B\left(T_{1}^{\lambda}\right)$. It follows that

$$
\begin{aligned}
0 \leq \lambda_{1}(M \backslash \Omega) & \leq \lambda_{1}\left(\Omega_{\lambda}\right) \\
& \leq \frac{\int_{\Omega_{\lambda}}\left|\nabla \varphi_{\lambda}\right|^{2} d M}{\int_{\Omega_{\lambda}}\left|\varphi_{\lambda}\right|^{2} d M} \\
& =\frac{\int_{T_{1}^{2}}^{T_{\lambda}^{\lambda}}\left|x_{\lambda}^{\prime}(r)\right|^{2} v(r) d r}{\int_{T_{1}^{\lambda}}^{T_{\lambda}^{\lambda}}\left(x_{\lambda}(r)\right)^{2} v(r) d r} \\
& =\frac{-\int_{T_{1}^{\lambda}}^{T^{\lambda}}\left(v(r) x_{\lambda}^{\prime}(r)\right)^{\prime} x_{\lambda}(r) d r}{\int_{T_{1}^{\lambda}}^{T_{\lambda}^{\lambda}}\left(x_{\lambda}(r)\right)^{2} v(r) d r} \\
& =\lambda .
\end{aligned}
$$

Since $\lambda$ is an arbitrary positive constant, $\lambda_{1}(M \backslash \Omega)=0$. This proves the part (a) of our theorem.

If the condition in (b) is satisfied, then from Theorem 2.1 for any $\lambda>\frac{a^{2}}{4}$ there exists a nontrivial oscillatory solution $x_{\lambda}(t)$ of $(2.1)$ on $\left[T_{0},+\infty\right)$ with $v(t)$ defined as in the beginning of the proof. Thus there exist two numbers $T_{1}^{\lambda}$ and $T_{2}^{\lambda}$ in $\left[T_{0},+\infty\right)$ such that $T_{1}^{\lambda}<T_{2}^{\lambda}$ and $x_{\lambda}\left(T_{1}^{\lambda}\right)=x_{\lambda}\left(T_{2}^{\lambda}\right)=0$, and $x_{\lambda}(t) \neq 0$ for any $t \in\left(T_{1}^{\lambda}, T_{2}^{\lambda}\right)$. Write $r(x)=\operatorname{dist}(x, p), \varphi_{\lambda}(x)=x_{\lambda}(r(x))$ and $\Omega_{\lambda}=B\left(T_{2}^{\lambda}\right) \backslash B\left(T_{1}^{\lambda}\right)$. It follows that

$$
\begin{aligned}
\lambda_{1}(M \backslash \Omega) & \leq \lambda_{1}\left(\Omega_{\lambda}\right) \\
& \leq \frac{\int_{\Omega_{\lambda}}\left|\nabla \varphi_{\lambda}\right|^{2} d M}{\int_{\Omega_{\lambda}}\left|\varphi_{\lambda}\right|^{2} d M} \\
& =\frac{\int_{T_{1}^{\lambda}}^{T^{\lambda}}\left|x_{\lambda}^{\prime}(r)\right|^{2} v(r) d r}{\int_{T_{1}^{\lambda}}^{T_{\lambda}^{\lambda}}\left(x_{\lambda}(r)\right)^{2} v(r) d r} \\
& =\lambda .
\end{aligned}
$$

Since $\lambda$ is an arbitrary positive constant larger than $\frac{a^{2}}{4}$, so $\lambda_{1}(M \backslash \Omega) \leq \frac{a^{2}}{4}$. This proves the part (b) of our theorem. 
Corollary 3.2. Let $M$ be a complete, noncompact Riemannian manifold with Ricci curvature satisfying

$$
\operatorname{Ric}(x) \geq-(n-1) \frac{\frac{1}{4}-c}{1+r^{2}(x)}
$$

outside some compact subset, where $r(x)$ is the distance function from a fixed point in $M$ and $c \in\left[0, \frac{(n+1)}{2(n-1)}\right]$. Then $\lambda_{1}(M \backslash \Omega)=0$ for any compact set $\Omega$ in $M$.

Proof. It follows from [CGT, Theorem 4.9] that the volume of the manifold is infinite and grows polynomially. So the conclusion follows directly from Theorem 3.1 .

The following corollary extends results due to Pinsky $[\mathrm{P}]$, Osserman $[\mathrm{O}](n=2)$ and Gage $[\mathrm{G}]$ (arbitrary $n$ ). The last assertion is actually contained in [G] Corollary 5.5. The referee has called our attention to the fact that the full result is contained in $(2.19)$ of $[\mathrm{T}]$.

Corollary 3.3. Let $M$ be a complete, noncompact Riemannian manifold with volume satisfying

$$
V(r) \leq C e^{a r}
$$

where $r(x)$ is the distance function from a fixed point in $M$ and $C$, a are some positive constants. Then $\lambda_{1}(M) \leq \frac{a^{2}}{4}$. In particular, if Ricci curvature is bounded below by some negative constant $-(n-1) k^{2}$ outside some compact subset, then $\lambda_{1}(M) \leq \frac{(n-1)^{2} k^{2}}{4}$.

Proof. If $V(M)<+\infty$, it follows from [CY, Proposition 9] that $\lambda_{1}(M)=0$. If $V(M)=+\infty$, from Theorem 3.1 we know $\lambda_{1}(M) \leq \frac{a^{2}}{4}$. Thus we have proved the first part.

If $\operatorname{Ric}(x) \geq-(n-1) k^{2}$ for any $x \in M \backslash B_{R}(o)$, where $B_{R}(o)$ is some geodesic ball with radius $R$ and center $o$, then from the proof of Heintze-Karcher's comparison theorem [HK] applied to $\partial B_{R_{1}}(o) \backslash C_{1}$ where $R_{1}>R$ and $C_{1}$ is the cut locus of 0 , we have

$$
V(r) \leq C_{n} e^{(n-1) k}
$$

So this time $a=(n-1) k$ and the conclusion follows.

Remark 3.1. The bounds obtained in Theorem 3.1 can be attained in the case of space forms. In (a) the volume condition can be replaced by subexponential growth in the sense that

$$
\lim _{r \rightarrow+\infty} \frac{\log \operatorname{vol}(B(r))}{r}=0 .
$$

By using the Ricci model introduced in [CrY] (see also [CGT]) we can obtain some volume information from the Ricci curvature which can lead to estimates of first eigenvalue. Here we give some special results. The following corollary follows from Theorem 3.1 and a straightforward calculation of the volume.

Corollary 3.4. Let $M=\left(R^{n}, d s^{2}\right)$ with $d s^{2}=d r^{2}+g^{2}(r) d \theta^{2}$ where $d \theta^{2}$ is the standard metric on the sphere $S^{n-1}$ and $g(t)$ is a $C^{2}$ function on $[0,+\infty)$ satisfying 
$g(t)>0$ as $t>0$ and $g(0)=0, g^{\prime}(0)=1$. Assume that

$$
\varlimsup_{t \rightarrow+\infty} \frac{\log \int_{0}^{t} g^{n-1}(s) d s}{t}=a<+\infty ;
$$

then $\lambda_{1}(M) \leq \frac{a^{2}}{4}$

In the above corollary, if we choose $g(r)=r e^{r+\sin r}$, then

$$
\varlimsup_{t \rightarrow+\infty} \frac{\log \int_{0}^{t} g^{n-1}(s) d s}{t} \leq \varlimsup_{t \rightarrow+\infty} \frac{\log \left[e^{(n-1)(t+1)} \cdot \frac{1}{n} \cdot t^{n}\right]}{t}=n-1,
$$

so we have $\lambda_{1}(M) \leq \frac{(n-1)^{2}}{4}$. This time the Ricci curvature satisfies

$$
\operatorname{Ric}(x)=-(n-1) \frac{g^{\prime \prime}(r(x))}{g(r(x))}=-(n-1)\left[\sin r(x)+(1+\cos r(x))^{2}\right] \geq-5(n-1),
$$

and we can only get the estimate $\lambda_{1}(M) \leq \frac{25(n-1)^{2}}{4}$ which is not so good. Combining the analysis above and Remark 4.2 in [CGT] we obtain

Corollary 3.5. Let $M$ be a complete, noncompact Riemannian manifold with Ricci curvature satisfying

$$
\operatorname{Ric}(x) \geq-(n-1)\left[\sin r(x)+(1+\cos r(x))^{2}\right]
$$

where $r(x)$ is the distance function from a fixed point in $M$. Then $\lambda_{1}(M) \leq \frac{(n-1)^{2}}{4}$

\section{Some applications}

I. In this section we give some applications of our eigenvalue estimates to geometric problems. The first application is a generalization of a theorem in [AdC].

Theorem 4.1. Let $x: M^{n} \rightarrow \bar{M}^{n+1}$ be an isometric immersion of complete Riemannian manifold with infinite volume into an oriented, complete Riemannian manifold. Assume $x$ has constant mean curvature $H$, ind $M<+\infty$ and the volume of $M$ has subexponential growth. Then there exists a positive constant $r_{0}>0$ such that for all $r \geq r_{0}$

$$
H^{2} \leq-\frac{1}{n} \inf _{M \backslash B(r)} \overline{\operatorname{Ric}}(N) .
$$

Proof. From [FC] we see that if ind $M<+\infty$, then there exist a compact set $K \subset M$ and a positive function $u$ on $M$ such that

$$
0=L u=\Delta u+\|B\|^{2} u+\overline{\operatorname{Ric}}(N) u \quad \text { on } M \backslash K .
$$

We can find a positive constant $r_{0}$ such that $K \subset B\left(r_{0}\right)$ and from Theorem 3.1 $\lambda_{1}(M \backslash B(r))=0$ for any $r \geq r_{0}$. By Lemma 2.3 of [AdC] we have

$$
\begin{aligned}
0 & =\lambda_{1}(M \backslash B(r)) \geq \inf \left(-\frac{\Delta u}{u}\right) \\
& \geq \inf _{(M \backslash B(r))}\left(\|B\|^{2}+\overline{\operatorname{Ric}}(N)\right) \\
& \geq \inf _{(M \backslash B(r))}\left(n H^{2}+\overline{\operatorname{Ric}}(N)\right) .
\end{aligned}
$$

Since $H$ is constant, we have proved our theorem. 
Remark 4.1. $V(M)=+\infty$ is automatically satisfied when the mean curvature of $M$ is bounded and the ambient space $\bar{M}^{n+1}$ has bounded geometry, i.e. sectional curvature bounded from above and injective radius bounded from below by a positive constant. This was proved in [Fr, Theorem 1 in Appendix].

Theorem 4.2. Let $x: M^{n} \rightarrow \bar{M}^{n+1}$ be an isometric immersion of complete Riemannian manifold with infinite volume into an oriented, complete Riemannian manifold. Assume $x$ has constant mean curvature $H$, ind $M<+\infty$ and the volume of $M$ satisfies $\operatorname{vol}(B(r)) \leq C e^{a r}$ when $r \geq 0$ for some constant $C$ and $a$. Then there exists a positive constant $r_{0}>0$ such that for all $r \geq r_{0}$

$$
H^{2} \leq \frac{a^{2}}{4 n}-\frac{1}{n} \inf _{M \backslash B(r)} \overline{\operatorname{Ric}}(N)
$$

In particular, if $\overline{\operatorname{Ric}}(N) \geq \frac{a^{2}}{4}$, then $M$ has to be minimal. If $\operatorname{Ric}_{M} \geq-(n-1) k^{2}$ outside a compact set, then

$$
H^{2} \leq \frac{(n-1)^{2} k^{2}}{4 n}-\frac{1}{n} \inf _{M \backslash B(r)} \overline{\operatorname{Ric}}(N)
$$

Proof. The proof of first claim is parallel to that of Theorem 4.1 and the rest can be easily seen.

Corollary 4.3. In the above theorem, when the volume of $M$ has subexponential growth and $\bar{M}^{n+1}=H^{n+1}(-1)$, then $H^{2} \leq 1$.

II. If the growth of volume of $M$ is specified and $M$ is immersed in a ball of some euclidean space, then the radius of the ball is bounded below by a number depending on the volume growth and the mean curvature of the immersion. More specifically,

Theorem 4.4. If $M^{n}$ has subexponential volume growth and $f(M) \subset B(o, \rho) \subset$ $R^{n+k}$ for some $o \in M$ and $\rho>0$, then

$$
\sup _{x \in M}|H(x)|>\frac{1}{\rho} .
$$

If $\operatorname{vol}(B(r)) \leq C e^{a r}$ and $\rho<\frac{2 \sqrt{n}}{a}$, then

$$
\sup _{x \in M}|H(x)|>\frac{1}{\rho} .
$$

Proof. Notice that for any $C^{2}$ function $f: R^{n+k} \rightarrow R^{1}$, the Laplacian of the restriction of $f$ to $M$ is given by

$$
\Delta_{M} f=\operatorname{tr}_{M}\left(\bar{D}^{2} f\right)+n\left\langle\mathbf{H}, \nabla_{R^{n+k}} f\right\rangle_{R^{n+k}}
$$


where $\mathbf{H}$ is the mean curvature vector. Assume that $\sup _{x \in M}|H(x)| \leq \frac{1}{\rho}$. Define $u(x)=\rho^{2}-r^{2}(x)$, where $r(x)$ is the distance between $x$ and $o$. Then

$$
\begin{aligned}
\Delta_{M} u(x) & =-2 n+n\left\langle\mathbf{H},-2 r(x) \nabla_{R^{n+k}} r(x)\right\rangle_{R^{n+k}} \\
& \leq-2 n+2 n|H(x)| \cdot r(x) \\
& =-\frac{2 n}{\rho}[\rho-r(x)] \\
& \leq-\frac{2 n}{\rho} \cdot \frac{1}{2 \rho}\left[\rho^{2}-r^{2}(x)\right] \\
& \leq-\frac{n}{\rho^{2}} u .
\end{aligned}
$$

It follows that $\lambda_{1}(M) \geq \frac{n}{\rho^{2}}$. Now the conclusion follows from Theorem 3.1 and Remark 3.2.

The question of Calabi [EK] asks: is there a complete minimal surface in $R^{3}$ which can be contained in some ball? Theorem 4.4 says that if a minimal surface is contained in some ball, the volume growth can't be too slow. We would like to conclude this paper with the following remark.

Remark 4.2. Our method to prove Theorem 4.4 can be applied in more general cases. It can be seen from the use of eigenvalue estimate results in this paper and the computations of the Laplacians of some appropriate functions. Since the idea is similar, we refer the interested readers to $[\mathrm{Z}]$ for some more detailed computations.

\section{REFERENCES}

[AdC] Alencar, H. and do Carmo, M.P., Hypersurfaces of constant mean curvatures with finite index and volume of polynomial growth, Arch. Math. 60 (1993), 489-493. MR 94a:53087; MR 96e: 53071

[CGT] Cheeger, J., Gromov, M. and Taylor, M., Finite propagation speed, kernel estimates for functions of the Laplace operator, and the geometry of complete Riemannian manifolds, J. Diff. Geometry 17 (1982), 15-53. MR 84b:58109

[CrY] Cheeger, J. and Yau, S.T., A lower bound for the heat kernel, Comm. Pure Appl. Math. 34 (1981), 465-480. MR 82i:58065

[CY] Cheng, S.Y. and Yau, S.T., Differential equations on Riemannian manifolds and geometric applications, Comm. Pure. Appl. Math. 28 (1975), 333-354. MR 52:6608

[EK] Eells, J. Jr., Kobayashi, S., Problems in differential geometry, In:, Proc. of US-Japan Seminar on differential geometry. Kyoto 1965, 167-177.

[F] Fite, W.B., Concerning the zeros of the solutions of certain differential equations, Trans. Amer. Math. Soc. 19 (1918), 341-352.

[FC] Fischer-Colbrie, D., On complete minimal surfaces with finite Morse index in threemanifolds, Invent. Math. 82 (1985), 121-132. MR 87b:53090

[Fr] Frensel, K.R., Stable complete surfaces with constant mean curvature, Bol. Soc. Bras. Mat. 27 (1996), 1-17. MR 98c:53068

[G] Gage, M.E., Upper bounds for the first eigenvalue of the Laplace-Beltrami operator, Indiana Univ. Math. J. 29 (1981), 897-912. MR 82b:58095

[HK] Heintze, E. and Karcher, H., A general comparison theorem with applications to volume estimates for submanifolds, Ann. Sci. École Norm. Sup. 11 (1978), 451-470. MR 80i:53026

[O] Osserman, R., Bonnesen style isoperimetric inequalities, Amer. Math. Monthly 86 (1979), 1-29. MR 80h:52013

[P] Pinsky, M., The spectrum of the Laplacian on a manifold of negative curvature I, J. Diff. Geometry 13 (1978), 87-91. MR 80g:58049

[T] Taylor, M., $L^{p}$-estimates on functions of the Laplace operator, Duke Math 58 (1989), 773-793. MR 91d:58253 
[W] Wong, J.S.W., Oscillation and nonoscillation of solutions of second order linear differential equations with integrable coefficients, Trans. Amer. Math. Soc. 144 (1969), 197-215. MR 40:4536

[Z] Zhou, D., Laplace inequalities with geometric applications, Arch. Math. 67 (1996), 50-58. MR 98b:53051

impa, Estrada Dona Castorina, 110-Jardim Botanico 22460-320 Rio de Janeiro, BRAZIL

E-mail address: manfredo@ impa.br

Department of Mathematics, Shandong University, Jinan, Shandong 250100, China 